\title{
Influencia de las diferentes alturas de tacón en la marcha. Estudio goniométrico
}

\author{
VILLARROYA, A.; COLOMA VILLACAMPA, S. Y LOITI \\ Escuela Universitaria de Ciencias de la Salud. \\ Unipersidad de Zaragoza.
}

\section{RESUMEN}

En este estudio se ha pretendido analizar las variaciones goniométricas en las articulaciones de la cadera, rodilla y tobillo, caminando descalzo y con tacón de 2 y 4 centímetros de altura, en dos grupos de mujeres $\sin$ patología y con edades comprendidas entre los 20 y 24 años: el primero acostumbradas a caminar con zapato de tacón alto ( 9 casos) y el segundo acostumbrado a caminar con calzado plano (21 casos). Para ello se ha utilizado un sistema telemétrico de medida: «Sistema Telemétrico Biológico de MIE Medical Rechearch LTD». Se ha comprobado que existen diferencias importantes en la forma individual de llevar zapato de tacón alto, que varían con la experiencia. La máxima flexión de cadera, que se produce en la fase de oscilación, disminuye conforme aumenta la altura del tacón en las personas acostumbradas al mismo, mientras que aumenta en las no acostumbradas. La extensión máxima de la rodilla disminuye conforme aumenta el tacón en todas las personas estudiadas, es decir, tanto si tienen experiencia como si no la tienen, mientras que la flexión máxima se mantiene. A nivel del tobillo, la adaptación al tacón se produce de forma muy dispar aunque llama la atención la exageración de la flexión del mismo, en las personas acostumbradas a llevar tacón, cuando caminan descalzas.

Palabras clave: Marcha, goniometría, tacón.

\section{SUMMARY}

By this study it is pretended to analyze the goniomethrical variations of hip, knee and ankle articulations, walking bare foot and with a heel-piece of 2 and $4 \mathrm{cmt}$., in two groups of women with no pathology and with ages between 20 and 24 years: the first group those used to walking with a high heel-piece ( 9 cases), and the rest used to walking with flat shoes (2l cases). To do this it has been used a telemethric measurement system: "MIE Medical Research LTD. Biological Telemethrical System". We have confirmed that there some differences in each way of wearing a high heel-piece shoe, depending on the experience. The maximum hip flexure, produced during the oscilation period, decreases while the heel-piece is higher in those women used to it, on the other hand it increases in those not used to. The maximum knee extension decreases while the heel-piece is higher in all women studied, that is, weather they are to or not, while the maximum flexion is maintained. In relation with the ankle the adaptability to the heel-piece is showed in a very dispair way, although it is significant the exageration of the flexion of that, in women used to ware high heel-piece shoes, when walking bare foot.

Key words: March, goniometry, ankle.

\section{INTRODUCCIÓN}

Aunque el uso del calzado fue adoptado muy pronto en Europa (los griegos utilizaban las esparteñas, los romanos adoptaron los coturnos cuya suela, más gruesa, permitía a los actores parecer más altos), el desequilibrio anteroposterior del calzado no aparece en Europa hasta Luis XIV. En el siglo XVIII es cuando se pusieron de moda $^{3}$ los tacones similares a los de aguja posteriores.

La moda puede influir mucho en el tipo de zapato; hoy se busca bastante la comodidad y se tiene a llevar calzado plano aunque se siguen llevando zapatos de tacón alto.

Algunos estudios se han centrado en analizar las variaciones biomecánicas que se producen cuando se camina con tacón alto. Entre ellos destacan los de OpilaCorreira en $1990^{7,8}$; pero la literatura sobre el tema no es demasiada, por lo que nos ha parecido interesante realizar un estudio cinemático de la extremidad inferior durante la marcha con diferentes alturas de tacón en dos grupos de mujeres; uno de ellos acostumbrado a llevar tacón alto y el otro acostumbrado a caminar con calzado plano, y analizar las variaciones existentes en cada grupo con las diferentes alturas de tạcón y también las diferencias entre ambos grupos.

\section{MATERIAL}

El estudio se ha realizado con un grupo de treinta mujeres, sin patología, de edades comprendidas entre veinte y veinticuatro años. Se ha hecho una pequeña división, teniendo encuenta que nueve de ellas usaban zapatos de tacón alto frecuentemente $(2$ ó $4 \mathrm{~cm}$ ) y las restantes prácticamente nunca habían llevado zapato de tacón alto.

Para registrar el movimiento de las articulaciones, el material empleado ha sido un telegoniómetro: Sistema Telemétrico Biológico de MIE Medical Research LTD. Es un aparato que consta de ocho canales formado por una unidad de transmisión y una unidad de recepción. A 
la unidad de trasmisión van conectados unos goniómetros así como un sensor que actuaba como desencadenante determinando el inicio del movimiento cuando se levantaba el talón del suelo. El aparato está conectado a una pantalla de ordenador que permite ver gráficamente las señales recogidas en las distintas articulaciones: cadera, rodilla y tobillo.

El ordenador empleado para el manejo de los datos ha sido un Macintosh SE; los programas empleados:

- Stat view, como programa estadístico.

- Excel, para los gráficos.

- McPaint 2.0, para elaborar las tablas.

\section{MÉTODO}

Los goniómetros electrónicos se colocaron en las articulaciones del miembro inferior derecho, conectados por medio de un cable a la unidad de transmisión que se llevaba en la cintura sujeta con un cinturón. La actividad realizada era recogida por el receptor conectado al ordenador en cuya pantalla aparecía el movimiento articular.

\section{La colocación de los goniómetros fué:}

- Cadera: El centro del goniómetro colocado en el borde superoexterno del trocánter mayor, una rama hacia cóndilo femoral externo y la otra hacia la espina ilíaca anterosuperior.

- Rodilla: El centro del goniómetro colocado en el cóndilo femoral externo, una rama del goniómetro dirigida hacia el centro del trocánter mayor y la otra hacia el maléolo externo.

- Tobillo: El centro del goniómetro colocado en el maléolo externo, una rama dirigida hacia la cabeza peroneal y la otra hacia la cabeza del quinto metatarsiano.

El estudio se realizó mediante tres pruebas con diferente altura de tacón cada una de ellas, la primera prueba se realizó con el individuo descalzo, la segunda con un zapato de tacón de dos centímetros y la tercera con cuatro centímetros (el tacón se midió por su parte anterior). Cada prueba consistió en caminar durante diez segundos en línea recta, sobre una superficie llana, repitiéndose tres veces con cada altura, con el fin de obtener un valor medio y conseguir así unos resultados más fiables. Bajo el talón se colocó un sensor para registrar cada momento en que esta zona del pie apoyaba y que servía de desencadenante para comenzar el registro del movimiento, en el momento que el talón abandonaba el suelo por primera vez.

Todas las personas vestían pantalón corto para que las prominencias óseas fueran fácilmente localizables y así facilitar la colocación de los goniómetros, que eran fijados a cada segmento por medio de velcro y de esparadrapo, consiguiendo con ésto registrar unos movimientos más fiables y dando más seguridad a la persona que realizaba la prueba. El sensor iba sujeto a la planta del pie o al zapato, según cada prueba, por medio de cinta de doble cara. Colocado todo el material y previa realización de la prueba, se les pidió que caminaran durante un período de tiempo con el fin de familiarizarse con los aparatos y comprobar que ningún cable initerfería o impedía la correcta realización del movimiento.

Una vez recogidas las señales fueron pasadas a un programa de ordenador (DSP), donde se podían analizar y se podía ver su representación gráfica en la pantalla. Hallamos los valores angulares máximos, mínimos y el rango de movimiento de cada una de las articulaciones estudiadas, comparándolos entre las diferentes alturas del tacón, así como entre las personas que estaban acostumbradas a llevar tacón y las que no.

Tras reunir todos estos datos, se introdujeiron en el ordenador y mediante un programa estadístico, stat view 512 , se manejaron los resultados. Par ver si las diferencias entre los valores de las personas acostumbradas y las no acostumbradas a llevar tacón eran significativas, se utilizó el test de Mann Whitney, que es un test no paramétrico (ya que la distribución de los valores no era normal o paramétrica); para la comparación de los valores obtenidos entre las tres alturas se utilizó el test de Friedmann (que también es un test no paramétrico) y para especificar más, se aplicó un test de Wilcoxon entre cada dos alturas.

En todos los casos se ha considerado como datos significativos los que tienen una probabilidad menor de 0,05 .

\section{RESULTADOS}

Hemos realizado el estudio en cada uno de lфs grupos y posteriormente su comparación.

\section{Acostumbrados}

En la tabla I se muestran los valores medios y desviaciones standard de los parámetros medidos en las personas acostumbradas a llevar zapáto de tacón alto, señalando si las diferencias de estos valores entre las tres alturas estudiadas son estadísticamente significativas.

Respecto a la máxima flexión de cadera apenas muestra diferencias llevando zapato de $2 \mathrm{cms}$. de tacón y cuando caminan descalzas; mientras que al elevar el tacón a $4 \mathrm{cms}$. la flexión disminuye siendo la diferencia estadísticamente significativa (gráfica 1).

La media del rango de cadera es ligeramente mayor cuando andan descalzas, mientras que con zapato de 2 y $4 \mathrm{cms}$. de tacón apenas hay diferencia. No hay significación en estos datos (gráfica 1).

Respecto a la flexión máxima de rodilla, què corresponde a la fase de oscilación, se observa que la media de los valores es ligeramente menor al caminar descalzas que en la marcha con 2 y $4 \mathrm{cms}$. de tacón (gráfica 2).

La máxima extensión de rodilla, se produce en el comienzo de la fase de doble apoyo. La mayor extensión se da con $2 \mathrm{cms}$. de tacón, sin embargo al caminar con $4 \mathrm{cms}$. aparece una flexión de rodilla; la diferencia entre ambos valores es significativa, habiendo una diferencia de $4,6^{\circ}$ de promedio (gráfica 2 ). 


\section{TABLA I}

MEDIAS (ENGRADOS) Y DESVIACIONES STANDARD DE LOS PARÁMETROS MEDIDOS EN LAS PERSONAS ACOSTUMBRADAS A LLEVAR ZAPATO DE TACÓN LATO, INDICANDO SI LAS DIFERENCIAS DE LOS VALORES ENTRE LAS 3 ALTURAS DE TACÓN SON SIGNIFICATIVAS MEDIANTE ${ }^{*} p=0.05 y^{\star \star} p=0.01$

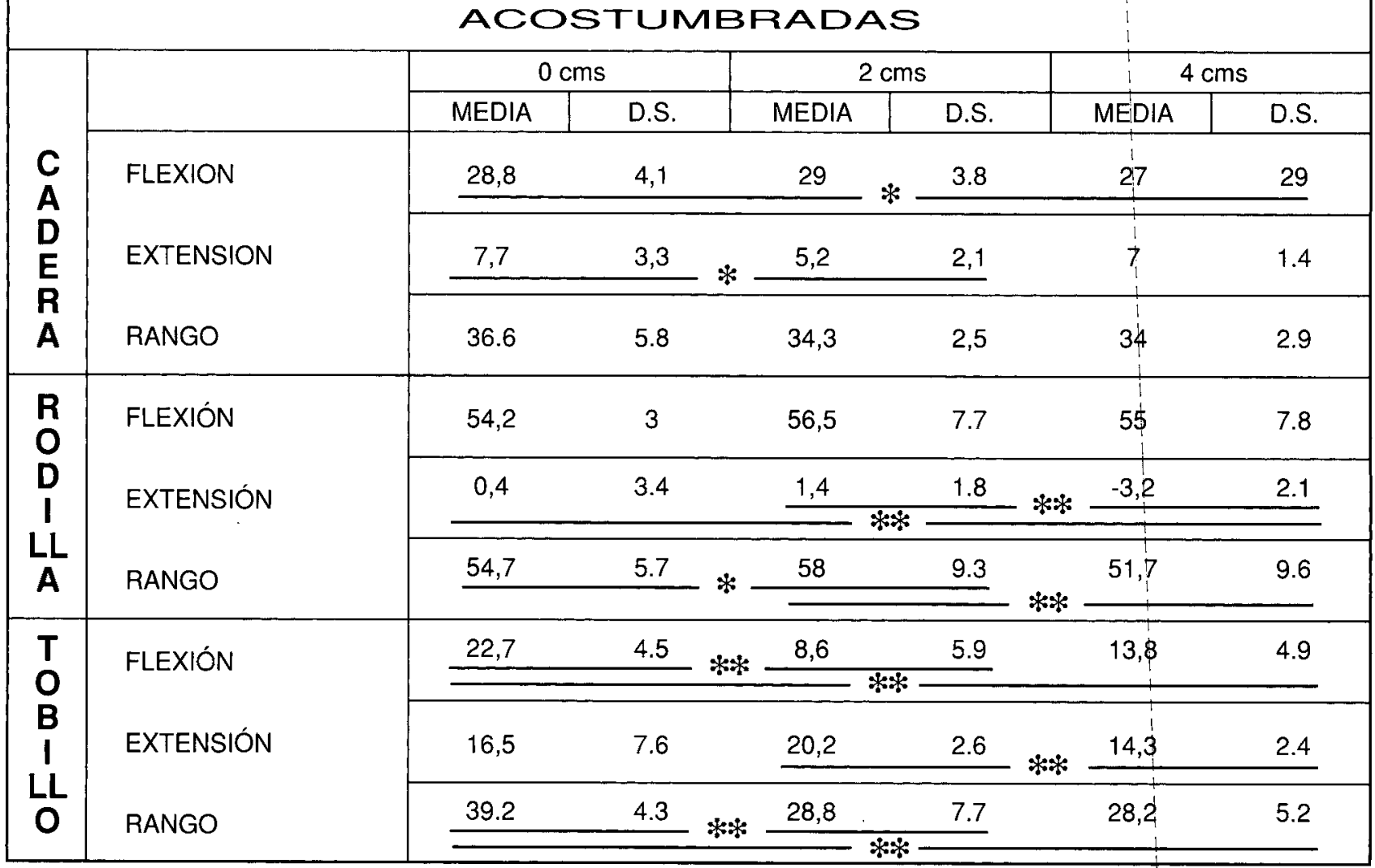

El rango de movimeinto de rodilla es mayor al caminar con $2 \mathrm{cms}$. de tacón, sin embargo disminuye muy significativamente al aumentar la altura del mismo hasta $4 \mathrm{cms}$., siendo en este momento cuando hay un menor rango de movimiento (gráfica 2).

En cuanto al tobillo, la flexión dorsal es máxima cuando caminan descalzas y mínima con $2 \mathrm{cms}$. de tacón. Al aumentar la altura a $4 \mathrm{cms}$. la flexión dorsal aumenta con rescpecto a los $2 \mathrm{cms}$., siendo las diferencias muy significativas (gráfica3).

No hay diferencias significativas en la flexión plantar o extensión entre la marcha descalzas y con $2 \mathrm{cms}$. de tacón, y sin embargo disminuye significativamente al llevar $4 \mathrm{cms}$ (gráfica 3).

El rango de movimiento del tobillo disminuye a medida que aumenta la altura del tacón. Siendo significativa la diferencia existente entre la marcha y con $2 \mathrm{cms}$. En la marcha con 2 y $4 \mathrm{cms}$. de tacón no hay apenas diferencia de rango (gráfica 3).

\section{No acostumbradas:}

En la tabla II se muestran las medias y desviaciones standard de los parámetros medidos en las personas no acostumbradas a llevar zapáto de tacón alto, señalando si las diferencias de estos valores entre las tres alturas estudiadas son estadísticamente signifícativas.

La flexión de cadera aumenta gradualmente con la altura del tacón, siendo estadísticamente significativa la diferencia entre la marcha con $2 \mathrm{cms}$. 'de tacón y $4 \mathrm{cms}$. Sin embargo los valores medios de la extensión de cadera disminuyen con la altura del tacón, sin que exista significación en estos datos (gráfica 1).

El rango de cadera aumenta proporcionalmente con la altura, pero muy ligeramente, con una probabilidad entre los diferentes valores đe 0,06 (grậfica 1).

La flexión máxima de rodilla no sufre 'variaciones significativas con las diferentes alturas del calzado (gráfica 2).

La máxima extensión de rodilla, se dà con zapato de 2 cms. y la menor extensión con $4 \mathrm{cms}$. 'siendo estadísticamente muy significativa la diferencia entre 2 y $4 \mathrm{cms}$. de tacón (gráfica 2).

El menor rango de movimiento de rodilla se produce al llevar zapáto con $4 \mathrm{cms}$. y el mayor con $2 \mathrm{cms}$, siendo significativa la diferencia entre estas alturas (gráfica 2). 


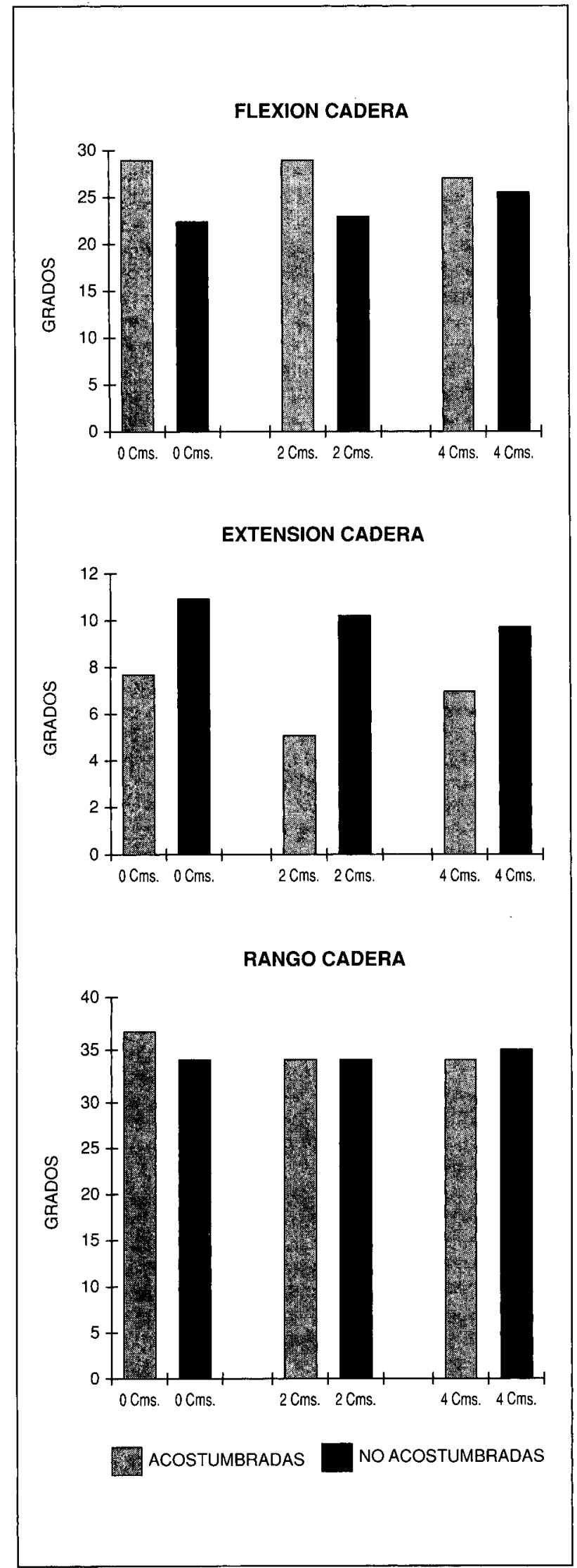

Gráfica 1: Valores de la máxima flexión, máxima extensión y rango de movimiento de la cadera durante la marcha con las 3 alturas de tacón, en personas acostumbradas y no a llevar tacón.

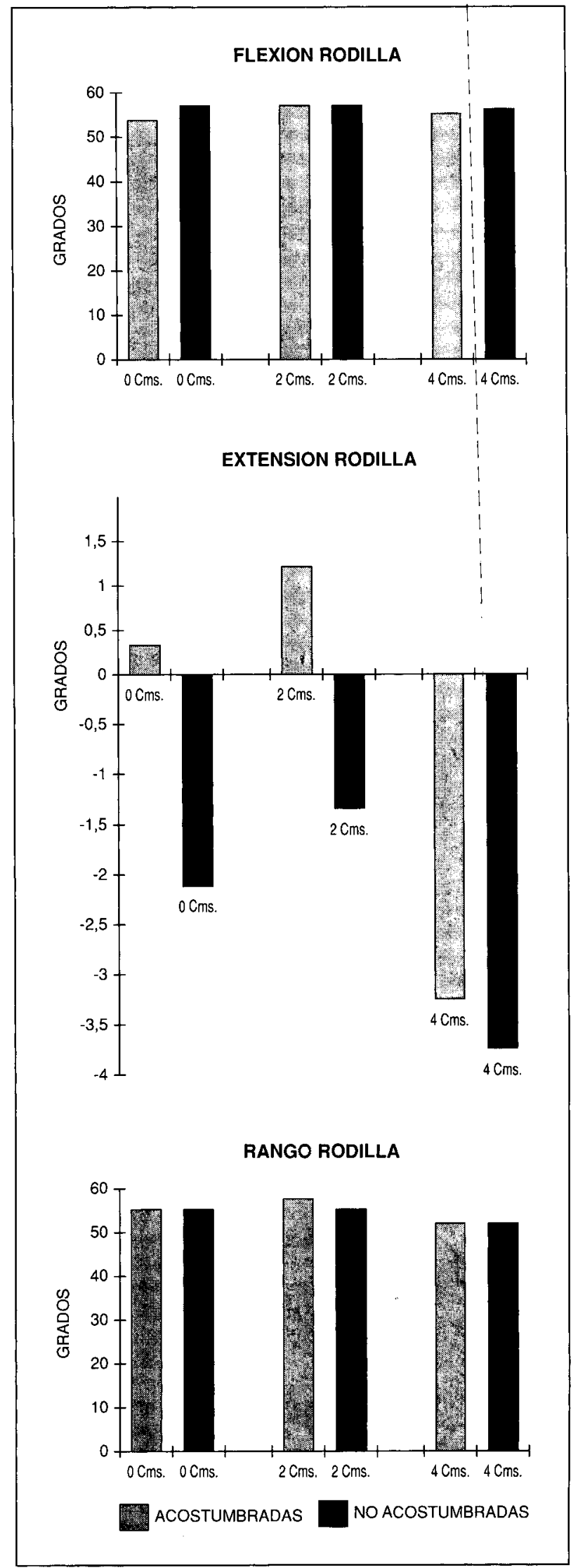

Gráfica 2: Valores de la máxima flexión, máxima extensión y rango de movimiento de la rodilla durante la marcha con las 3 alturas de tacón, en personas acostumbradas y no a llevar tacón. 


\begin{tabular}{|c|c|c|c|c|c|c|c|}
\hline \multicolumn{8}{|c|}{$\begin{array}{l}\text { TABLA } 2 \\
\text { MEDIAS (ENGRADOS) Y DESVIACIONES STANDARD DE LOS PARÁ } \\
\text { EN LAS PERSONAS ACOSTUMBRADAS A LLEVAR ZAPATO DE } \\
\text { INDICANDO SI LAS DIFERENCIAS DE LOS VALORES ENTRE LAS } 3 \\
\text { SON SIGNIFICATIVAS MEDIANTE * } \mathrm{p}=0.05,{ }^{\star *} \mathrm{p}=0.01 \mathrm{y} \\
\text { NO ACOSTUMBRADAS }\end{array}$} \\
\hline \multirow{5}{*}{$\begin{array}{l}\text { C } \\
\text { A } \\
\text { D } \\
\text { E } \\
\mathbf{R} \\
\text { A }\end{array}$} & \multirow{4}{*}{$\begin{array}{l}\text { FLEXION } \\
\text { EXTENSION }\end{array}$} & \multicolumn{2}{|c|}{$0 \mathrm{cms}$} & \multicolumn{2}{|c|}{$2 \mathrm{cms}$} & \multicolumn{2}{|c|}{$4 \mathrm{cms}$} \\
\hline & & MEDIA & D.S. & MEDIA & D.S. & MEDIA & D.S \\
\hline & & 23,1 & 5.3 & \multicolumn{2}{|l|}{24,1} & 26 & 5.3 \\
\hline & & 10,9 & 1.4 & 10,3 & 2.1 & 9,8 & 1.8 \\
\hline & RANGO & 34 & 5.2 & 34,6 & 4.9 & 35,8 & 5.3 \\
\hline \multirow{3}{*}{$\begin{array}{l}R \\
\mathbf{O} \\
\mathbf{D} \\
\mathbf{I} \\
\mathrm{LL} \\
\mathbf{A}\end{array}$} & FLEXIÓN & 56,9 & 10 & 56,6 & 10.4 & 55,9 & 7.6 \\
\hline & EXTENSIÓN & $-2,1$ & 4.9 & -1.3 & 3.4 & $-3,7$ & 4.6 \\
\hline & RANGO & 54,8 & 5.9 & 55,4 & 9.7 & 52,1 & 5.9 \\
\hline \multirow{3}{*}{$\begin{array}{c}T \\
0 \\
B \\
I \\
\text { LL } \\
\mathbf{O}\end{array}$} & FLEXIÓN & 15,8 & 6.7 & 16,3 & 7.1 & 15,4 & 6 \\
\hline & EXTENSIÓN & 8,3 & 4.4 & 8,7 & 4.8 & 10,4 & 3.2 \\
\hline & RANGO & 24,1 & 6.2 & 25 & 6.1 & 25,8 & 4.5 \\
\hline
\end{tabular}

La flexión dorsal del tobillo es muy similar en los tres casos, aunque la media está un poco aumentada con respecto a las demás al llevar un tacón de $2 \mathrm{cms}$. La diferencia no es significativa (gráfica 3 ).

La flexión plantar del tobillo es similar cuando caminan descalzas y con $2 \mathrm{cms}$ de tacón y la media aumenta, sin significación al elevar el tacón a $4 \mathrm{cms}$ con respecto al de 2 cms (gráfica 3).

El rango de movimiento del tobillo es ligeramente mayor cuando căminan descalzas y es prácticamente igual con las otras dos alturas de tacón (gráfica 3).

\section{Comparación acostumbradas - no acostumbradas}

En la tabla III se muestran las medias de los parámetros medios en las personas acostumbradas y en las no acostumbradas a llevar zápato de tacón alto, en las tres alturas de tacón, señalando si las diferencias de estos valores son estadísticamente significativas.

La flexión de cadera es mayor en las personas acostumbradas, con las tres alturas de tacon, respecto a las personas no acostumbradas. Esta diferencia es significativa solo cuando caminan descalzas (gráfica 1).

La extensión de cadera es significativamente mayor en las personas no acostumbradas, con las tres alturas de tacón (gráfica 1).

No hay diferencia importante en el rango de movimiento de cadera entre los grupos que suelen llevar tacón y los que no, con variación de las alturas del mismo (gráfica1).

La flexión máxima de rodilla es significativamente mayor en las personas no acostumbradas cuando caminan descalzas, siendo muy parecida con las otras alturas, mientras que la extensión de rodilla es mayor en las acostumbradas en las tres alturas de tacón, siendo estas diferencias estadísticamente significativas cuando caminan descalzas y con $2 \mathrm{cms}$ (gráfica 2).

El rango de movimiento de rodilla es muy similar en ambos grupos cuando caminan descalzas y con $4 \mathrm{cms}$., sin embargo es mayor en las personas acostumbradas con $2 \mathrm{cms}$. de tacón aunque sin significación estadística (gráfica 2). 


\begin{tabular}{|c|c|c|c|c|}
\hline \multicolumn{5}{|c|}{$\begin{array}{l}\text { TABLA } 3 \\
\text { MEDIAS (EN GRADOS) DE LOS PARAMETROS MEDIDOS EN LAS PERSONAS } \\
\text { ACOSTUMBRADAS Y NO ACOSTUMBRADAS A LLEVAR TACÓN ALTO, } \\
\text { MEDIANTE \#p }=0.05, \# \#=0.01 \text { y \#\# } p=0.00 \\
\text { SEÑALANDO SI LAS DIFERENCIAS ENTRE LOS VALORES SON ESTADÍSTICAMENTE SIGNIFICATIVAS, } \\
\text { COMPARACIÓN ACOSTUMBRADAS NO ACOSTUMBRADAS }\end{array}$} \\
\hline \multirow{10}{*}{$\begin{array}{l}\mathbf{C} \\
\mathbf{A} \\
\mathbf{D} \\
\mathbf{E} \\
\mathbf{R} \\
\mathbf{A}\end{array}$} & & ACOSTUMBRADAS & NO ACOSTUMBRADAS & PROBABILIDAD \\
\hline & FLEX. 0 cms. & 28,8 & 23,1 & \#\# \\
\hline & FLEX. $2 \mathrm{cms}$. & 29 & 24,1 & \\
\hline & FLEX. $4 \mathrm{cms}$. & 27 & 26 & \\
\hline & EXT. $0 \mathrm{cms}$ & 7,7 & 10,9 & $\#$ \\
\hline & EXT. $2 \mathrm{cms}$. & 5,2 & 10,5 & \#\#\# \\
\hline & EXT. $4 \mathrm{cms}$. & 7 & 9,8 & $\# \#$ \\
\hline & RANGO $0 \mathrm{cms}$. & 36,6 & 34 & \\
\hline & RANGO $2 \mathrm{cms}$. & 34,3 & 34,6 & \\
\hline & RANGO $4 \mathrm{cms}$. & 34 & 35,8 & \\
\hline \multirow{9}{*}{$\begin{array}{c}R \\
0 \\
D \\
I \\
L L \\
A\end{array}$} & FLEX. $0 \mathrm{cms}$. & 54,2 & 56,9 & $\# \#$ \\
\hline & FLEX. 2 cms. & 56,5 & 56,6 & \\
\hline & FLEX. $4 \mathrm{cms}$. & 55 & 55,9 & \\
\hline & EXT. $0 \mathrm{cms}$. & 0,4 & $-2,1$ & $\#$ \\
\hline & EXT. 2 cms. & 1,4 & $-1,3$ & $\#$ \\
\hline & EXT. $4 \mathrm{cms}$. & $-3,2$ & $-3,7$ & \\
\hline & RANGO $0 \mathrm{~cm}$. & 54,7 & 54,8 & \\
\hline & RANGO $2 \mathrm{~cm}$. & 58 & 55,4 & \\
\hline & RANGO $4 \mathrm{cms}$. & 51,7 & 52,1 & \\
\hline \multirow{9}{*}{$\begin{array}{c}T \\
0 \\
B \\
1 \\
L L \\
0\end{array}$} & FLEX. 0 cms. & 22,7 & 15,8 & \#\# \\
\hline & FLEX $2 \mathrm{cms}$. & 8,6 & 16,3 & \#\# \\
\hline & FLEX $4 \mathrm{cms}$. & 13,8 & 15,4 & \\
\hline & EXT. 0 cms. & 16,5 & 8,3 & \#\# \\
\hline & EXT. 2 cms. & 20,2 & 8,7 & \#\#\# \\
\hline & EXT. 4 cms. & 14,3 & 10,4 & \#\# \\
\hline & RANGO $0 \mathrm{~cm}$ & 39,2 & 24,1 & \#\#\# \\
\hline & RANGO $2 \mathrm{~cm}$ & 28,8 & 25 & \\
\hline & RANGO $4 \mathrm{~cm}$ & 28,2 & 25,8 & \\
\hline
\end{tabular}

Por último, en lo que respecta al tobillo, la flexión dorsal es significativamente mayor en las personas acostumbradas en relación a las que no lo están cuando caminan descalzas, mientras que con 2 y $4 \mathrm{cms}$. de tacón ocurre lo contrario, aunque en estos últimos casos la diferencia solo es significativa con $2 \mathrm{cms}$. de tacón (gráfica 3).

La flexión plantar es significativamente mayor en las personas acostumbradas en las tres alturas (gráfica 3).

Se aprecian diferencias importantes en el rango de movimiento del tobillo; es también mayor en las personas acostumbradas, aunque solo hay significación al caminar descalzas (gráfica 3).

\section{DISCUSIÓN}

La aumentada flexión plantar causada por la elevación del talón, el desplazameinto del centro de gravedad adelante y la inestabilidad causada por la reducción del área de apoyo, producen una alteración general en la biomecánica de la marcha, la cual debe ser acomodada en la cinemática de los miembros inferiores, pelvis y columna.

Esta acomodación se produce de forma distinta según la costumbre que tengan las personas de andar con zapátos de mayor o menor tacón, puesto que cada individuo adopta un patrón de marcha acorde a sus características propias constitucionales o del calzado que lleva habitualmente, entre otras. 
Durante la fase de oscilación de la marcha, en la que se produce la máxima flexión de cadera y rodilla, en las personas que están acostumbradas a llevar zapáto de tacón, se produce una disminución de la flexión de la cadera conforme aumenta el tacón, mientra que en las no acostumbradas aumenta.

Esta disminución de la flexión de cadera que se observa en las acostumbradas, se debe probablemente a que esta máxima flexión se produce en la fase de oscilación $y$ al tener el otro miembro inferior apoyado en alto por el tacón, no necesitan flexionar tanto en la triple flexión que se da en el miembro inferior oscilante para que no choque el pie en el suelo. Esto coincide con los estudios realizados por Opila Correira ${ }^{7}$, en los que se ven los mismos resultados. Sin embargo, en el grupo con menor experiencia el aumento de flexión de cadera que se observa, pensamos que puede ser debido a un intento algo exagerado, al no tener costumbre, de evitar que el tacón choque con el suelo.

Llama la atención que esta flexión de cadera es significativamente mayor en las personas que suelen llevar zapato de tacón, cuando caminan descalzas, probablemente porque, aunque disminuye esta flexión conforme aumenta el tacón, están acostumbradas a caminar con esta flexión de cadera y la mantienen aún cuando caminan sin tacón; sin embargo las que no suelen llevarlo, al ir descalzas tienen una flexión menor que las del otro grupo.

La flexión de rodilla que hemos encontrado durante esta fase, no varía con las diferentes alturas de tacón en ninguno de los dos grupos los que no está de acuerdo con los trabajos de Opila Correira ${ }^{7}$ quien encuentra una ligera disminución de esta flexión en las personas experimentadas conforme aumenta el tacón.

En el inicio de la fase de apoyo es cuando se produce la máxima extensión de la rodilla y la máxima flexión dorsal del tobillo. La extensión de rodilla en ambos grupos disminuye con el aumento del tacón, es decir, caminan más en flexión de rodilla, siendo más significativas estas diferencias en las no acostumbradas sobre todo al pasar de los 2 a los $4 \mathrm{cms}$. de tacón. Estos resultados coinciden con otros estudios anteriores como los de Murray, Opila Correir ay otros ${ }^{4,6,7,8}$, ya que al aumentar el tacón, aumenta la flexión plantar del pie, que aumenta la flexión de la rodilla. Esta flexión de rodilla, como indica Joseph ${ }^{5}$, hace que haya un aumento y prolongación de la actividad de cuádriceps con tacón alto.

Comparándo los dos grupos, vemos que la disminución de esta extensión, es mayor en el segundo o grupo de las no acostumbradas que en el primero; esta diferencia es significativa con un tacón de $2 \mathrm{cms}$., ya que las no acostumbradas flexionan más que las que lo están, compensando estas últimas el aumento de la altura del tacón sobre todo con la flexión de cadera y, como dice Opila Correira $^{8,9}$, con una hiperlordosis, lo contrairo a las experimentadas que suelen caminar con una lordosis rectificada ${ }^{8,9,1}$. Pero con $4 \mathrm{cms}$. aunque hay diferencias entre los valores, no son significativas, ésto se debe probablemente a que las que suelen llevar tacón, lo habitual es que lleven alrededor de 2 cms. más o menos, y por tanto se han adaptado a esa altura, pero al pasar a $4 \mathrm{cms}$. de altura actúan como no acostumbradas a esta nueva altura y aumentan la flexión de la rodilla, de forma semejante a las que no llevan habitualmente tacón. Por todo lo dicho, el rango de movimiento de rodilla disminuye con mayor altura de tacón ya que hemos visto que se mantiene la máxima flexión pero disminuye la extensión.

Respecto a la flexión dorsal del tobillo, no hay diferencias significativas en las personas que no suelen llevar tacón, ya que cada persona se comporta de una forma y los valores articulares son muy dispares. Lo que sí llama la atención es que estos valores son mayores que los de las personas que suelen llevar tacón, siendo significativa esta diferencia con $2 \mathrm{cms}$. de tacón; ésto se debe a que estas últimas personas apoyan la planta casi a la vez y realizan una flexión mucho menor. Sin embargo, cuando éstas van descalzas y tienen que llegar con el talón al suelo, exageran la flexión dorsal haciendo movimientos extraños, siendo significativamente mayor que en las no acostumbradas.

Al final de la fase de apoyo se produce la máxima extensión de la cadera, así como la máxima flexión plantar o extensión del tobillo.

En ambos grupos hay una tendencia a disminuir la extensión de la cadera conforme aumenta el tacón. Pero con una menor extensión en las acostumbradas que, como hemos comentado, caminan más en flexión de cadera cuando van con calzado plano. Así mismo el rango de movimiento de esta articulación no varía, ya que como ya hemos citado, también aumenta la flexión máxima con la altura del tacón, es decir, caminan con la cadera más flexionada pero con igual rango de movimiento.

La extensión del tobillo, en el grupo de las no acostumbradas, al igual que la flexión, es muy variada por lo que no hay diferencias significativas entre los valores de unas alturas y otras. Sin embargo, sí son significativas las diferencias de la extensión entre ambos grupos tanto caminando descalzas como con las otras dos alturas de tacón. Estos valores son mayores en las que suelen llevar tacón, ya que partiendo de la posición de extensión que tienen, debido al tacón, realizan una extensión para dar el impulso al miembro inferior, sin embargo las que no suelen llevar tacón, cuando lo llevan, realizan el mismo movimeitno de extensión que realizarían sin llevarlo; como dice Ducroquet ${ }^{3}$, apenas se dan impulso, intentando compensar esta falta de impulso con contorsiones pelvianas.

El rango de movimiento del tobillo, es muy parecido con 2 y $4 \mathrm{cms}$. de tacón en los dos grupos. Sin embargo las personas acostumbradas al tacón alto al ir descalzas tienen un rango de movimiento significativamente mayor que las otras, debido sobre todo a la exageración de la flexión dorsal del pie al apoyar el talón en el suelo.

\section{Por tanto podemos decir que:}

- Existen diferencias importantes en la forma individual de llevar zapáto de tacón alto, que varía con la experiencia.

- La máxima flexión de cadera, que se produce en la fase de oscilación, disminuye conforme aumenta la altu- 
ra de tacón en las personas acostumbradas al mismo, mientras que aumenta en las no acostumbradas.

- La extensión máxima de la rodilla disminuye conforme aumenta el tacón en todas las personas estudiadas, es decir, tanto si tienen experiencia como si no la tienen, mientras que la flexión máxima se mantiene.

- A nivel del tobillo la adaptación al tacón se produce de forma muy dispar aunque llama la atención la exageración de la flexión dorsal del mismo, en las personas acostumbradas a llevar tacón, cuando caminan descalzas.

\section{BIBLIOGRAFIA}

1. BENDIX T, SORENSON S.S, KLAUSEN K.

Lumbar curve, trunk muscles, and line of gravity with different heel heights. Spine. 9:223-7

2. DE LATEUR, B.I.: GIACONI, R.M.; QUESTAD, K.; KO, M. Y LEHMANN, J.F.:

Footwear and posture. Compensatory strategies for heel-height. Am.J. Phys. Med. Rehabil. Vol 5, Oct.: 246-254, 1991.

3. DUCROQUET, R.I. Y P.:

Marcha normal y patológica.

Editorial Toray Masson, 1982

4. GOLLNICK P.D, TIPTON C.M, KARPOVICH P.V.

Electrogoniometric study of walking on high heels. Res Q. 35: suppl:370-8. 1964

5. JOSEPH J.

The pattern of activity of some muscles in women walkingon high heels.

Ann. Phys. Med. 9:295-9. 1968.

6. MURRAY M.P, KORY R.C, SEPIC S.B.

Walking pattems of normal women.

Arch. Phys. Med. Rehabil. 51:637-50. 1970

7. OPILA CORREIA, K.A.:

Kinematics of high-heeled gait.

Arc. Phys. Med. Rehabil. Vol 71:304-310, 1990.

8. OPILA CORREIA, K.A.:

Kinematics of high-heeled gait with consideration for age and experience of wearers. Arch. Phys. Med. Rehabil. Vol 71: $905-$ $909,1990$.

9. OPILA CORREIA, K.A.:

Kinematics of high-heeled gait with consideration for age and experience of wearers. Arch. Phys. Med. Rehabil. Vol 71: 905$909,1990$.

10. OPILA-CORREIRA K.A, WAGNER S.S, SCHIOWITZ S, CHEN J.

Postural alignment in barefoot and high-heeled stance. Spine. 13: 542-7. 1988. 